\title{
State-of-Charge Balancing Control of a Battery Power Module for a Modularized Battery for Electric Vehicle
}

\author{
Seong-Chon Choi*, Jin-Yong Jeon**, Tae-Jung Yeo**, Young-Jae Kim**, Do-Yun Kim* and \\ Chung-Yuen Won ${ }^{\dagger}$
}

\begin{abstract}
This paper proposes a State-of-Charge (SOC) balancing control of Battery Power Modules (BPMs) for a modularized battery for Electric Vehicles (EVs) without additional balancing circuits. The BPMs are substituted with the single converter in EVs located between the battery and the inverter. The BPM is composed of a two-phase interleaved boost converter with battery modules. The discharge current of each battery module can be controlled individually by using the BPM to achieve a balanced state as well as increased utilization of the battery capacity. Also, an SOC balancing method is proposed to reduce the equalization time, which satisfies the regulation of a constant DC-link voltage and a demand of the output power. The proposed system and the SOC balancing method are verified through simulation and experiment.
\end{abstract}

Keywords: Battery power modules, State-of-charge, SOC balancing, Electric vehicles.

\section{Introduction}

Interest in eco-friendly vehicles has been increasing due to environmental problems; power conversion devices for Electric Vehicles (EVs) have therefore attracted an increasing amount of attention. EVs use battery packs as their sole energy source, and can operate without releasing pollutants. Thus, the motor for EVs needs to be supplied with energy from the battery while the EV is being driven, and the DC-link voltage should be regulated at a constant value [1]. Therefore, a DC-DC converter located between the battery and the inverter is required to transfer the charged energy of the battery to the motor and maintain a constant DC-link voltage. In particular, a step-up converter is essential because the voltage provided by the battery is lower than that of the DC-link [2].

Batteries should have a long life-cycle and a high energy capacity in order to extend the driving range and to ensure the safety of EVs. Therefore, a plurality of battery cells connected in series and parallel can be used for battery packs to provide high voltage and high capacity power. Meanwhile, the repetitive charge and discharge operation of battery packs can cause a State-Of-Charge (SOC) imbalance of the battery cells connected in series since the internal impedances and chemical characteristic of the battery cells differ. The SOC imbalance of the battery cells reduces the battery life and degrades battery energy utilization, sometimes leading to an explosion. To solve

$\dagger \quad$ Corresponding Author: College of Information and Communication Engineering, Sungkyunkwan Univ., Korea. (woncy@skku.edu)

* College of Information and Communication Engineering, Sungkyunkwan Univ., Korea. (chon8787@skku.edu)

** Materials R\&D Center, Samsung advanced institute of technology, Korea. (jywjw.jeon@samsung.com)

Received: March 23, 2015; Accepted: November 29, 2015 this problem, discharge and charge equalization methods have been introduced [3-9]. Conventional cell balancing methods can be considered to be either dissipative or energy recovery schemes. A dissipative balancing method has the advantages of requiring only a simple configuration of balancing circuit and has a low cost. However, it has a drawback in that the energy efficiency is low because the energy of a battery cell with a high SOC is dissipated through the resistor in parallel. On the other hand, the energy recovery balancing method has high energy efficiency because the energy is shifted from a battery cell with a high SOC to a battery cell with a low SOC by using capacitors and converters. However, these methods require a complicated configuration and have a high cost. A Battery Power Module (BPM) is another solution, which consists of battery cells with corresponding output converters $[8,9]$. BPMs effectively overcome the abovementioned battery imbalance issues and also address the nonlinear characteristic of the battery voltage profile. However, the energy recovery balancing method and BPM, equipped with a dedicated cell balancing circuit, have a low balancing current.

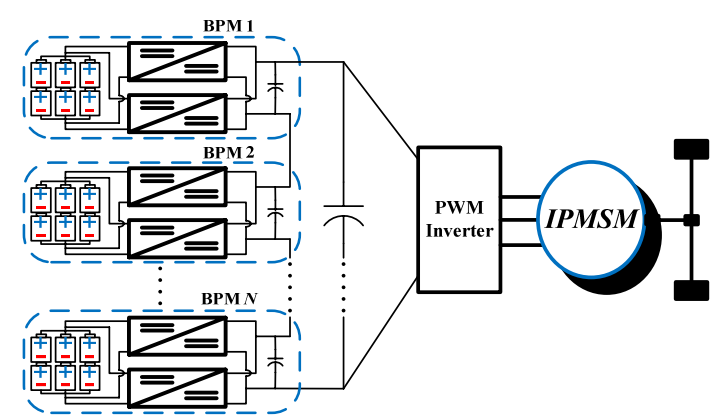

Fig. 1. Configuration of BPMs in EV. 
This paper proposes a SOC balancing control of BPMs that can be used to balance the difference of SOC values between the battery modules for $\mathrm{EV}$ without additional balancing circuits while maintaining the total regulated DC-link voltage. Fig. 1 shows the configuration of the $\mathrm{BPMs}$ in $\mathrm{EV}$, which are composed of a number of modularized DC-DC converters and battery modules. In [8], BPMs are connected to each battery cell. A large number of BPMs are needed because the battery pack for EV consists of a number of battery cells in series. This structure of BPMs is not suitable in EV application having battery pack with enormous battery cells due to complex circuits and control. Battery cells are modularized to avoid the above-mentioned problem. The BPM used in this paper uses a two-phase interleaved boost converter to reduce the ripple of the input/output current/voltage and to divide a relatively large current at the input side. The BPM is connected to the battery module, which has a nominal voltage of $24 \mathrm{~V}$ and a rated capacity of $200 \mathrm{Ah}$. The outputs of the BPM are connected in series so that a relatively high DC-link voltage can be obtained. A synchronous boost converter is used to obtain high efficiency by replacing the diode with a Metal Oxide Semiconductor Field Effect Transistor (MOSFET). To achieve a balanced battery state and to improve battery utilization, the discharge current of each battery module is individually controlled by using the BPMs. This paper also proposes a balancing method based on SOC with battery modules to reduce balancing time while maintaining DC-link voltage regulation.

Section 2 introduces the SOC estimation method used in this paper. Theoretical analysis of the proposed SOC balancing method using the BPMs is explained in detail in Section 3. The simulation and experimental results are presented in Section 4 and 5 to verify the system operation and the SOC balancing method.

\section{SOC Estimation}

The SOC information of each battery module is needed in order to implement the proposed balancing method with the module unit. SOC Estimation methods have been extensively studied in the literature $[10,11]$. The SOC estimation method in this paper is combined with a Coulomb counting method and battery modeling. The

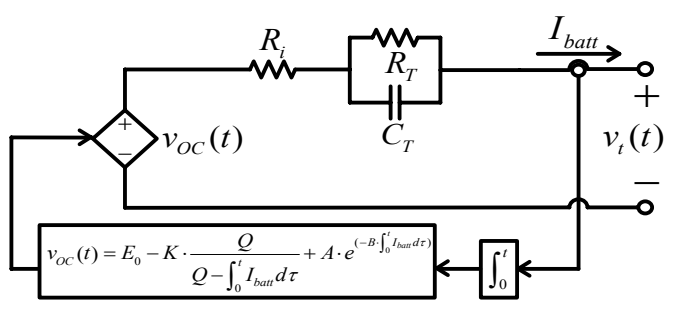

Fig. 2. Battery modeling coupled with the Shepherd model and the Thevenin model. battery modeling is coupled with the Shepherd model and the Thevenin model. The Shepherd model can describe the unique non-linear Open Circuit Voltage (OCV) waveform of the battery by using the parameters in the datasheet of the actual battery. Fig. 2 shows the battery modeling coupled with the Shepherd model and the Thevenin model. The Shepherd model which represents the electrochemical battery can be expressed as

$$
\begin{gathered}
v_{O C}(t)=E_{0}-K \cdot \frac{Q}{Q-\int_{0}^{t} I_{\text {batt }} d \tau}+A \cdot e^{\left(-B \cdot \int_{0}^{t} I_{\text {bat }} d \tau\right)} \\
E_{o}=E_{\text {Full }}+K+R_{i} \cdot I_{\text {batt }}-A \\
A=E_{\text {Full }}-E_{E x p}, B=\frac{3}{Q_{E x p}} \\
K=\frac{\left(E_{\text {Full }}-E_{\text {Nom }}+A\left(\exp \left(-B \cdot Q_{\text {Nom }}\right)-1\right)\right) \cdot\left(Q-Q_{\text {Nom }}\right)}{Q_{\text {Nom }}}
\end{gathered}
$$

where $v_{O C}(t)$ is the open circuit voltage, $E_{0}$ is the battery constant voltage, $K$ is the polarization voltage, $A$ is the exponential zone amplitude, $I_{b a t t}$ is battery current, and $B$ is the exponential zone time constant inverse of the battery module.

Specifications of the one cell of a lead-acid battery are described in Table 1. The battery module consists of the four lead acid battery packs connected in 2-series and in 2-parallel. The lead acid battery pack is composed of 6-cell in series. $E_{\text {Full }}$ is the full charged voltage of the battery module where two lead-acid battery packs are connected in series (i.e., one pack $=6$ cell $\times 2.15=12.9 \mathrm{~V}$, module $=$ $12.9 \times 2=25.8 \mathrm{~V}) . E_{E x p}$ is the exponential zone voltage of the battery module $(=24.6 \mathrm{~V})$, and $E_{N o m}$ is the nominal zone voltage of the battery module $(=24 \mathrm{~V}), Q$ is the total capacity of the battery module, which is $100 \mathrm{Ah}$ in order to adjust the time scale for the discharge curve. $Q_{\text {Nom }}$ is the capacity of the battery module in the nominal zone (=80Ah), $Q_{E x p}$ is the capacity in the exponential zone of the battery module ( $=20 \mathrm{Ah})$, and $R_{i}$ is the internal resistance of the battery module $(=0.04 \Omega)$.

The Thevenin battery model with parallel RC circuits is added to represent the behavior characteristic corresponding to the change in the transient load so that this model can express the physical properties of the battery. Finally, the battery terminal voltage $v_{t}(t)$ is given as

$$
v_{t}(t)=v_{O C}(t)-I_{b a t t}(t)\left[R_{i}+\left(C_{T} / / R_{T}\right)\right]
$$

where $R_{T}$ is the transient resistance and $C_{T}$ is the transient capacitance.

Table 1. Specifications of one cell of a lead-acid battery.

\begin{tabular}{c|c|c|c|c|c}
\hline Item & $\begin{array}{c}\text { Battery } \\
\text { affiliation }\end{array}$ & $\begin{array}{c}\text { Battery } \\
\text { capacity }\end{array}$ & $\begin{array}{c}\text { Nominal } \\
\text { voltage }\end{array}$ & $\begin{array}{c}\text { Full charged } \\
\text { voltage }\end{array}$ & $\begin{array}{c}\text { Internal } \\
\text { resistance }\end{array}$ \\
\hline Rating & Lead-acid & $100 \mathrm{Ah}$ & $2 \mathrm{~V}$ & $2.15 \mathrm{~V}$ & $0.013 \Omega$ \\
\hline
\end{tabular}




\section{Proposed SOC Balancing Method using BPMs}

\subsection{Basic concepts of the proposed SOC balancing}

If an inconsistency in the SOC values occurs between the battery modules, the utilization of the battery capacity decreases. Hence, it is essential to use BPMs to achieve SOC balancing for battery modules that have different SOC values. Fig. 3 shows the operational principle of SOC balancing for the battery modules using BPMs during discharge. The proposed SOC balancing method for the battery modules adjusts the battery discharge current of each battery module using BPMs.

Assuming that the power loss of each converter module is neglected, the related equations are obtained as

$$
\begin{gathered}
P_{B N}=P_{o N} \\
V_{B N} \cdot I_{B N}=V_{o N} \cdot I_{o}
\end{gathered}
$$

where $P_{B N}$ is the power of the $N$-th battery module, $P_{o N}$ is the output power of the $N$-th BPM, and $N$ is the number of modules.

The output voltage of the $N$-th BPM $V_{o N}$ will affect the discharge current of the $N$-th battery module $I_{B N}$ since the battery voltage of the $N$-th battery module $V_{B N}$ changes very little and the output current $I_{o}$ of all BPMs is the same. For this reason, $I_{B N}$ changes in accordance with the output voltage of the corresponding BPM. Consequently, the proposed method solves the inconsistency of the SOC values of the battery modules by increasing the output voltage of those BPMs that have a relatively higher remaining capacity and by reducing the output voltage of those BPMs that have a relatively lower remaining capacity during discharge. To solve the imbalance of the SOC values between the battery modules during charge, if the SOC value of the battery module is relatively higher than that of other battery modules, the output voltage of the

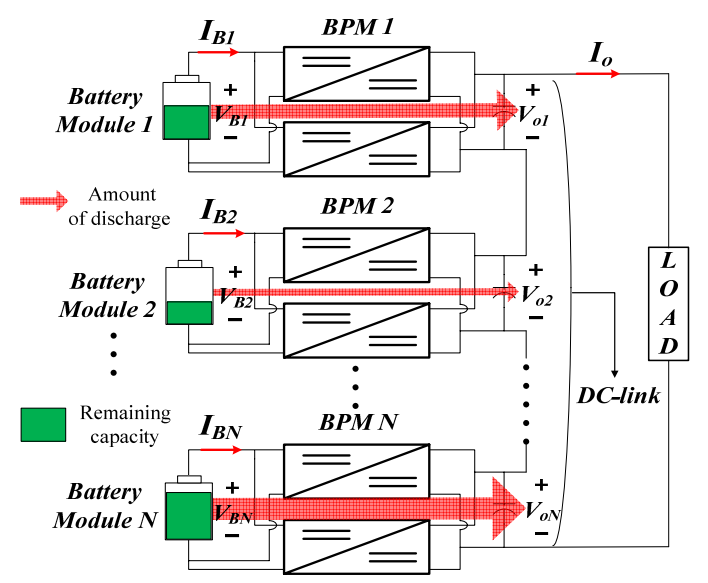

Fig. 3. Operational principle of SOC balancing for the using BPMs during discharge. corresponding BPM is decreased. If the SOC value of the battery module is relatively lower than that of other battery modules, the output voltage of the corresponding BPM is increased. Namely, the power of each battery module is proportional to the output voltage of the corresponding BPM, and its relationship can be expressed as

$$
P_{B 1}: P_{B 2}: \cdots: P_{B N}=V_{o 1}: V_{o 2}: \cdots: V_{o N}
$$

Thus, the SOC imbalance between battery modules is solved by controlling the output voltage of each BPM. Meanwhile, the step-up ratio of the interleaved converter is equal to the step-up of the boost converter, and it can be expressed as

$$
\frac{V_{o N}}{V_{B N}}=\frac{1}{1-d_{N}}
$$

where $d_{N}$ is the duty ratio of the single converter of the $\mathrm{N}$ th BPM. As a result, $I_{B N}$ can be derived as

$$
I_{B N}=\frac{I_{o}}{1-d_{N}}
$$

The average discharge current of each battery module can be expressed as

$$
I_{B 1}: I_{B 2}: \cdots: I_{B N}=\frac{1}{1-d_{1}}: \frac{1}{1-d_{2}}: \cdots: \frac{1}{1-d_{N}}
$$

\subsection{The proposed balancing method for battery module}

The SOC balancing method should satisfy the following three conditions.

- Achieve outstanding SOC balancing performance for battery modules

- Ensure fast balancing speed

- Maintain a constant and stable DC-link voltage

Fig. 4 shows a control flowchart for the proposed SOC balancing method. The SOC values of each battery module are checked to array the BPMs in order of the highest SOC value during discharge or in order of the lowest SOC during charge. Then, the difference between the highest and lowest SOC values of battery modules $S O C_{\text {diff }}$ is calculated. If $S O C_{\text {diff }}$ is more than the limit of the difference in the SOC values between battery modules $S O C_{\text {diff limit }}$, then $S O C_{\text {diff }}$ is equal to $S O C_{\text {diff limit. }}$ The output voltage reference values of the $i$-th BPM $V_{o i}{ }^{*}$ for SOC balancing are determined, considering the regulation of the DC-link voltage(i.e., $i=1,2,3 \cdots, N$ ). The output voltage of the $i$-th BPM $V_{o i}$ will be controlled so that it is proportional to the SOC value of the $i$-th battery module $S O C_{i}$; note that the battery module with a relatively higher SOC value is 


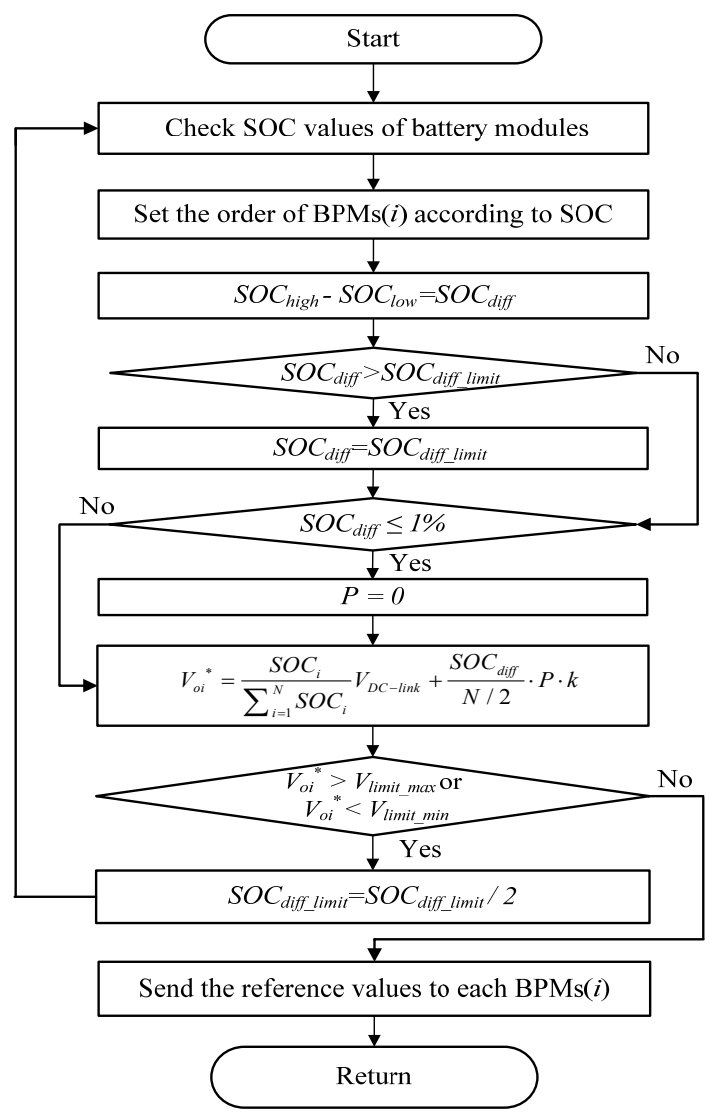

Fig. 4. Control flowchart of proposed balancing method.

required to have a relatively higher power to achieve SOC balancing. The relation between $V_{o i}$ and $S O C_{i}$ can be expressed as

$$
S O C_{1}: S O C_{2}: \cdots: S O C_{i}=V_{o 1}: V_{o 2}: \cdots: V_{o i}
$$

From (12), the ratio between $V_{o i}$ and $S O C_{i}$ is equal to the ratio between the sum of each $V_{o i}$ and the sum of each $S O C_{i}$. It is obtained as follows

$$
\frac{S O C_{i}}{V_{o i}}=\frac{S O C_{1}+S O C_{2}+\cdots+S O C_{i}}{V_{o 1}+V_{o 2}+\cdots+V_{o i}}
$$

In (13), the sum of each $V_{o i}$ is the DC-link voltage of the system; therefore, each $V_{o i}$ for SOC balancing is obtained as follows

$$
V_{o i}=\frac{S O C_{i}}{\sum_{i=1}^{N} S O C_{i}} \cdot V_{D C-l i n k}
$$

If $V_{o i}$ is determined to achieve the SOC balancing by using only (14), the equalization speed decreases as the balancing proceeds. To solve the above problem, the appropriate weight must be added to improve the SOC balancing speed. The weight is proportional to $S O C_{\text {diff }}$ in order to increase the balancing speed as the SOC imbalance increases, and it is reduced to alleviate fluctuation of the DC-link voltage as the number of BPM increases. The proportional constant value $P$ is also added to realize the improved balancing speed. Finally, each $V_{o i}{ }^{*}$ for SOC balancing is given by

$$
V_{o i}^{*}=\frac{S O C_{i}}{\sum_{i=1}^{N} S O C_{i}} V_{D C-\text { link }}+\frac{S O C_{d i f f}}{N / 2} \cdot P \cdot k
$$

where $k$ is the compensation coefficient value. As BPM increases, it becomes difficult to individually set the weight value of each BPM. Moreover, the sum of the weights that are applied to each module should be zero in order to maintain a constant DC-link voltage for the system. Therefore, $k$ is considered to allocate the weight to each BPM, and it can be expressed as

$$
k= \begin{cases}\frac{N}{2}-i & \left(\text { If } \frac{N}{2} \geq i\right) \\ 0 & \left(\text { If } \frac{N+1}{2}=i\right) \\ \frac{N}{2}+1-i & \left(\text { If } \frac{N}{2}<i\right)\end{cases}
$$

Each $V_{o i}{ }^{*}$ is utilized in the each voltage controller of BPMs, and then each BPM is controlled so that it has a different output voltage and discharge current in order to achieve the SOC balancing for the battery modules. Also, the DC-link voltage is regulated at a constant value because the sum of each $V_{o i}{ }^{*}$ does not exceed the predetermined DC-link voltage reference value $V_{D C \text {-link }}$ from (15). However, an undesired change in the order of BPMs occurs due to sensing noise because the SOC differences between the battery modules are almost similar at the end of SOC balancing. This means that the variation range of the output voltage for each BPM increases and causes unstable DC-link voltage. To solve this problem, a dedicated control is necessary. In this paper, if $S O C_{\text {diff }}$ is less than $1 \%, P$ is set to zero to minimize the variation range of the output voltage for each BPM.

If $V_{o i}{ }^{*}$ is larger than or smaller than the maximum output voltage reference $V_{\text {limit_max }}$ or the minimum output voltage reference $V_{\text {limit_min }}$, respectively, each $V_{o i}{ }^{*}$ is calculated again by reducing $S O C_{\text {diff_limit. }}$. When the rearranged $V_{o i}{ }^{*}$ is within the proper range, the master module sends each output voltage reference to the corresponding slave module.

\subsection{Control method of BPMs}

Fig. 5 shows the circuit and contol block diagram of the proposed system. The proposed system consists of three BPMs and extra modules can be installed to fulfill the capacity requirements for an inverter of the EV. The single BPM consists of two phase interleaved boost converter to 


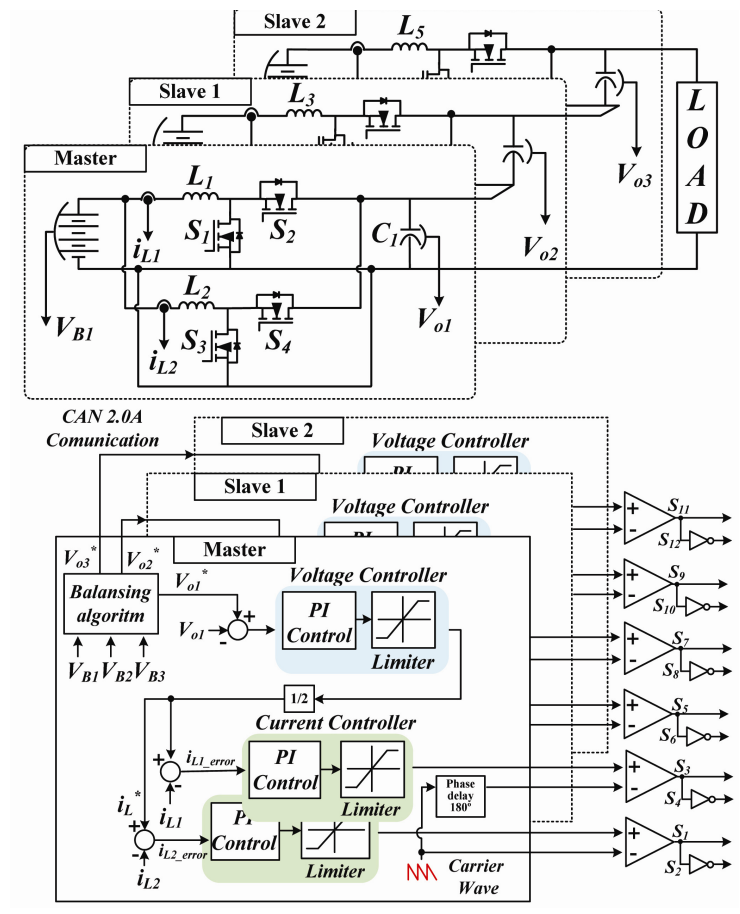

Fig. 5. Circuit and contol block diagram of the proposed system.

reduce the ripple of the voltage/current and to divide a relatively large input current. The synchronous rectification is used with the MOSFET on behalf of freewheeling diodes to improve the balancing efficiency. Each BPM is connected with its associated battery module and is independently controlled in accordance with the proposed SOC balancing method and status of the required load. The proposed system is divided into one master module and two slave modules. The master module transmits the output voltage reference generated by the proposed balancing algorithm after acquiring the SOC information of the slave battery modules. Here, the Controller Area Network (CAN) communication is used to obtain the SOC information of the slave battery modules and transfer the output voltage reference for each module by sharing a common CAN bus. The controller of each module uses the current inner loop for input current and the voltage outer loop for output voltage in the cascade. On the other hand, the one module consists of two converters in parallel, so an imbalance can occur in the current flowing through the respective converters. Thus, two converters are required for each of the current controllers. The output values for each controller are compared to the carrier signal, and the Pulse Width Modulation (PWM) signals are output from the comparators. As a consequence of utilizing a synchronous boost converter, if a PWM signal for the duty cycle $d_{N}$ operates switch $S_{1}$, a PWM signal for the duty cycle $1-d_{N}$ then operates switch $S_{2}$ with the adequate dead time. Here, two converters operate with the same carrier frequency, but with a phase delay. The $S_{3}$ and $S_{4}$ switches are shifted $180^{\circ}$ with respect to the $S_{1}$ and $S_{2}$ switches.

\section{Simulation Results}

Simulation results are provided to verify the proposed SOC balancing method and the operation of the system. The battery model combined with the Shepherd model and the Thevenin model was used. The SOC value of each battery module was estimated based on the Coulomb counting method. The boost inductor and the output capacitor of the rated capacity were designed considering the switching frequency at full load. The simulation parameters are shown in Table 2.

Fig. 6 shows the simulation of the key waveforms of the BPMs without the proposed SOC balancing method; note that the initial SOC values of the battery modules are master $=96 \%(25 \mathrm{~V})$, slave $1=98 \%(25.56 \mathrm{~V})$, and slave 2

Table 2. Simulation parameters of BPMs.

\begin{tabular}{c|c|c}
\hline Parameter & Value & Unit \\
\hline Rated power & 15.9 & $\mathrm{~kW}$ \\
\hline Inductance of boost inductor & 40 & $\mu \mathrm{H}$ \\
\hline Capacitance of DC-link capacitor & 8800 & $\mu \mathrm{F}$ \\
\hline DC-link voltage & 90 & $\mathrm{~V}$ \\
\hline Frequency & 25 & $\mathrm{kHz}$ \\
\hline Battery type & Lead-acid & - \\
\hline Battery capacity & 100 & $\mathrm{Ah}$
\end{tabular}

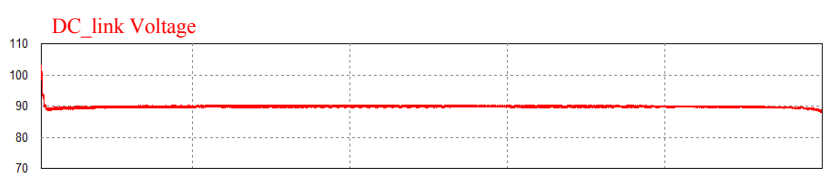

(a) DC-link voltage

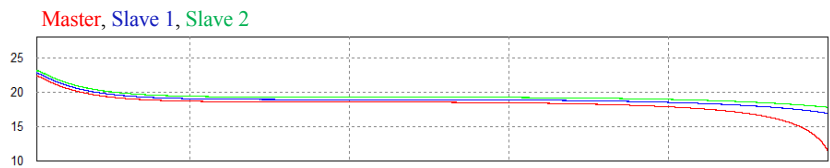

(b) Battery voltage

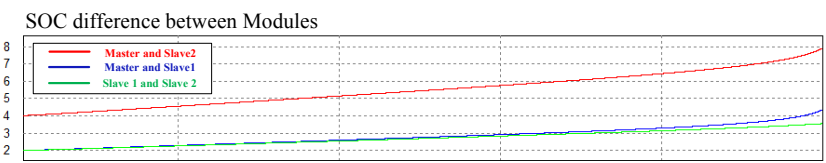

(c) SOC difference between the modules

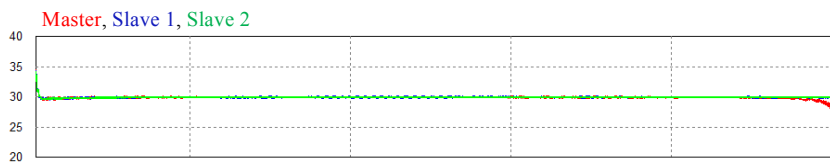

(d) Output voltage

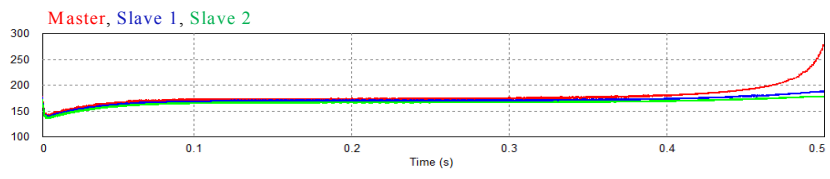

(e) Discharge current

Fig. 6. Simulation of key waveforms without the proposed SOC balancing method. 
$=100 \%(25.8 \mathrm{~V})$. Fig. $6(\mathrm{a})$ shows that DC-link voltage is maintained at $90 \mathrm{~V}$, but it is unstable because the output voltage of the master module is reduced. Fig. 6(b) and Fig. 6(c) show the battery voltage of each battery module and the SOC difference between battery modules, respectively, which do not converge and diverge greatly with time. Fig. 6(d) shows that the output voltage of each BPM is controlled to be the same value of 30V. In Fig. 6(d), the output voltage of the master module is reduced as the corresponding battery voltage falls below the cut-off region; note that the battery of the master module has the lowest initial SOC value. As the battery voltage is decreased, the discharge current increases as shown in Fig. 6(e) and the SOC imbalance therefore deteriorates gradually with reducing utilization of battery capacity. Fig. 7 shows the simulation of the key waveforms during the equalization with the proposed SOC balancing method. The DC-link voltage must be constant to ensure the motor operates in a stable manner. It can be confirmed that the DC-link voltage, which is the sum of each output voltage of the BPMs, is maintained at a constant value during the equalization as shown in Fig. 7(a). Fig. 7(b) shows the battery voltage of each battery module; note that the initial SOC values of the battery modules are the same as that

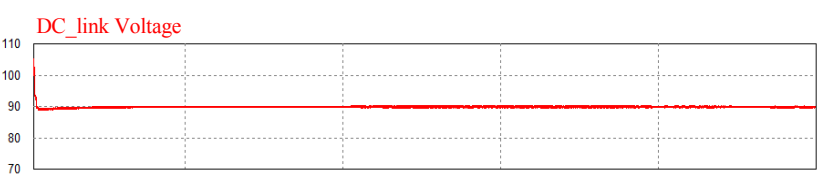

(a) DC-link voltage

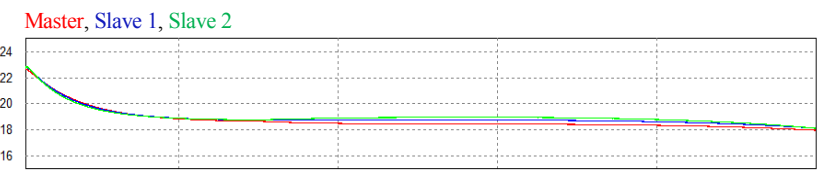

(b) Battery voltage

SOC difference between Modules

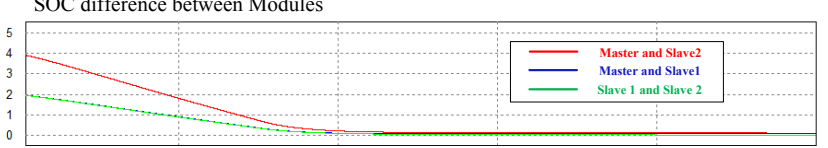

(c) SOC difference between the modules

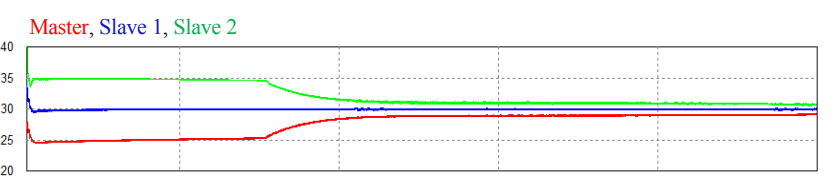

(d) Output voltage

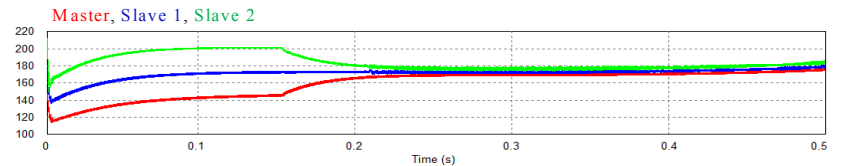

(e) Discharge current

Fig. 7. Simulation of key waveforms with the proposed SOC balancing method. shown in Fig. 6. The maximum $S O C_{\text {diff }}$ is defined as $4 \%$, where the SOC imbalance occurs between the battery modules as shown in Fig. 7(c). Fig. 7(b) and Fig. 7(c) show that the difference of battery voltage and SOC value between the battery modules decreases as balancing proceeds, respectively. Fig. 7(d) shows the output voltage of each BPM, which is proportional to the battery voltage and SOC value of each battery module. The discharge rate of the battery module for each BPM is proportional to the output voltage of each BPM as shown in Fig. 7(e).

\section{Experimental Results}

\subsection{SOC balancing results using BPMs}

The proposed SOC balancing method and the operation of the system are verified by making a prototype with three BPMs and implementing the experiment. The prototype of a single BPM is shown in Fig. 8. The parameters related to the test can be found in Table 2 . The battery module consists of the four lead acid batteries connected in 2-series and in 2-parallel. The nominal voltage and the rated capacity of the battery module are $24 \mathrm{~V}$ and $200 \mathrm{Ah}$, respectively. The SOC value of each battery module is estimated by using the Coulomb counting method and battery modeling.

Fig. 9 shows experimental waveforms of the BPMs, where the proposed SOC balancing algorithm is not applied (i.e., the initial SOC values of the battery modules are master $=95.5 \%(25.02 \mathrm{~V})$, slave $1=96.8 \%(25.25 \mathrm{~V})$, and slave $2=99.9 \%(25.95 \mathrm{~V}))$. The output voltage of each $\mathrm{BPM}$ is regulated at $30 \mathrm{~V}$ as shown in Fig. 9(a). Fig. 9(b) shows that the discharge current of each battery module is charged in accordance with the SOC value of the corresponding battery module (i.e., the lower the SOC value, the higher the discharge current). Thus, the master module with the lowest SOC value has the higher discharge current $(=57 \mathrm{~A})$ than the discharge current (=55A) of the slave 2 module with the highest SOC value. If the unbalanced battery voltage and discharge current

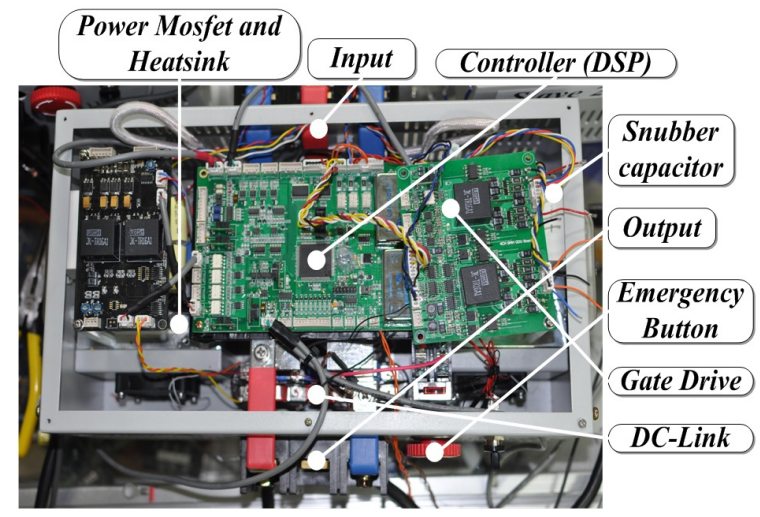

Fig. 8. Experimental prototype of the single BPM. 


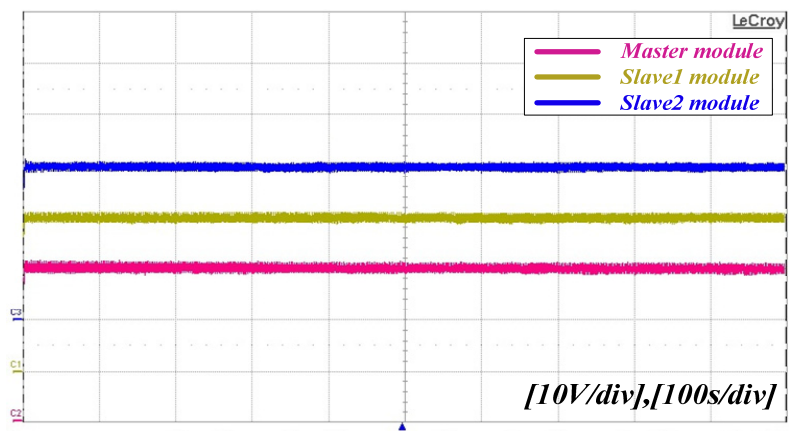

(a) Output voltage

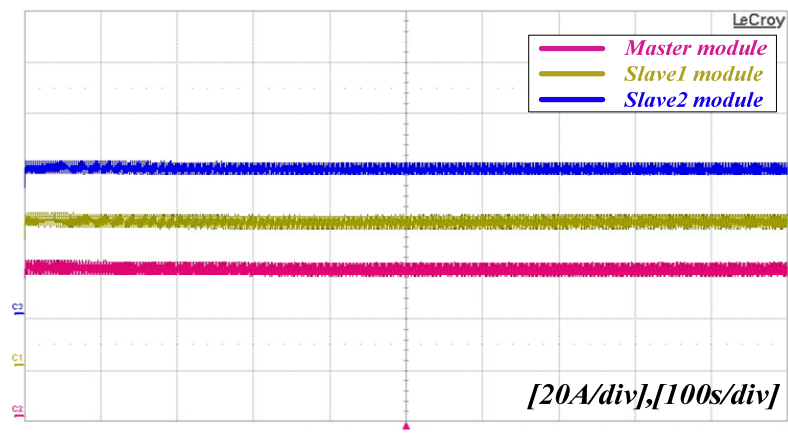

(b) Discharge current

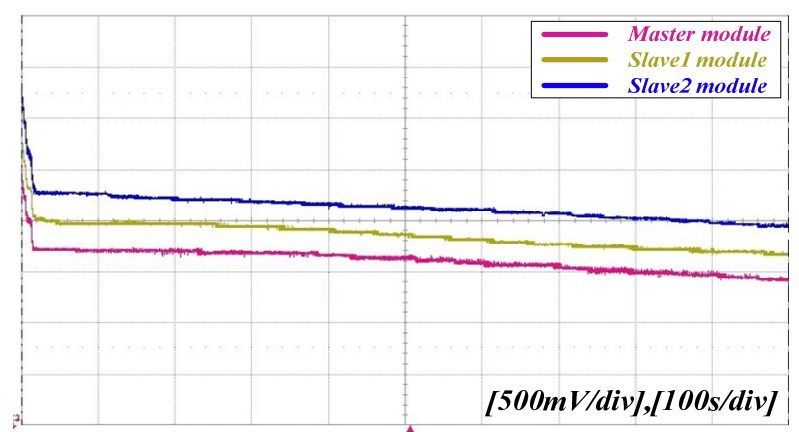

(c) Battery voltage

Fig. 9. Experimental waveforms without the proposed SOC balancing method.

between the battery modules increase, the difference in the discharge speed between the battery modules will increase with time. This phenomenon causes low utilization of the battery capacity because master module with the relatively high discharge speed will reach the cut-off region in advance. Fig. 9(c) shows the battery voltage of each battery module. Fig. 9(c) shows that the difference of the battery voltage between battery modules does not converge. $\mathrm{S} O C_{\text {diff }}$ gradually increases with time, which is $4.8 \%$ after 16.6 minutes.

Fig. 10 shows the experimental waveforms of the BPMs, where the proposed SOC balancing algorithm is applied (i.e., the initial SOC values of the battery modules are master $=96.5 \%(25.1 \mathrm{~V})$, slave $1=97.1 \%(25.35 \mathrm{~V})$, and slave $2=99.6 \%(25.9 \mathrm{~V}) . P$ is very closely related to the equalization time. If $P$ is increased, the balancing speed

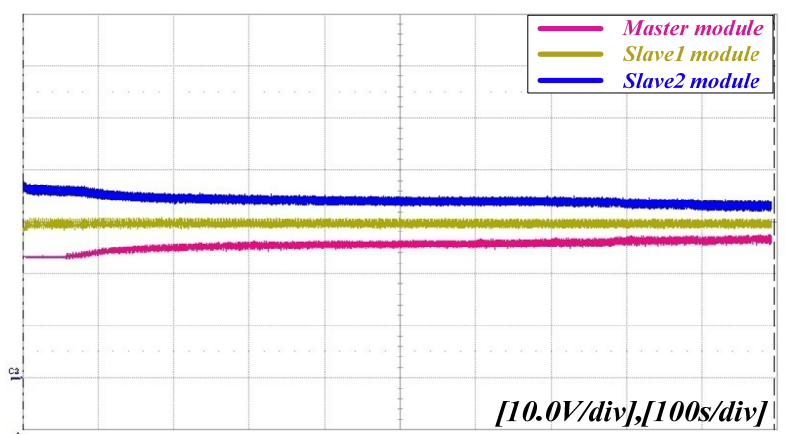

(a) Output voltage

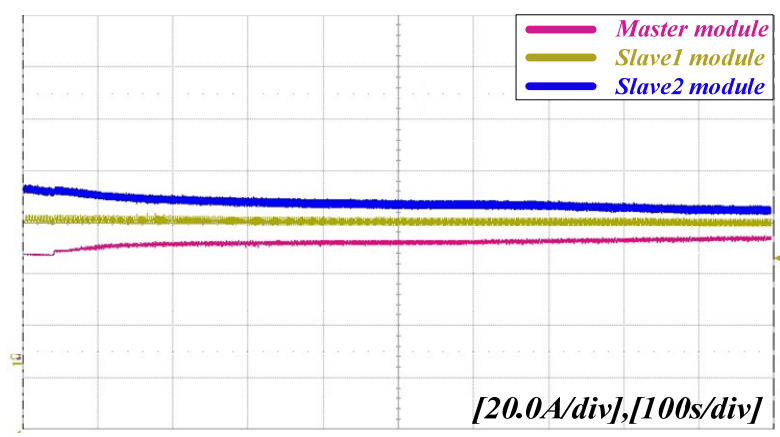

(b) Discharge current

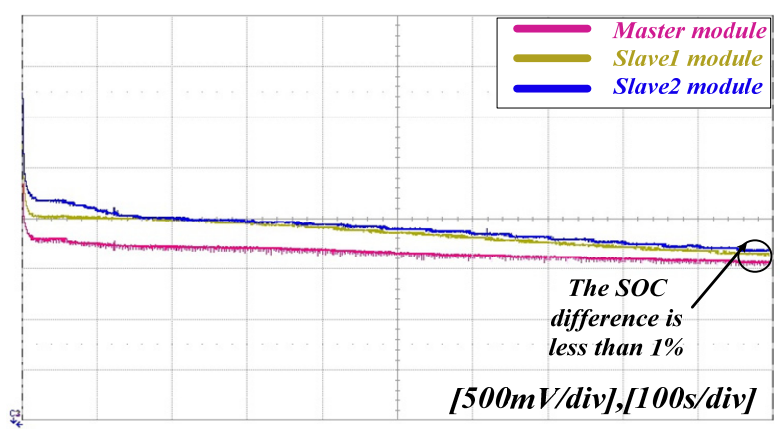

(c) Battery voltage

Fig. 10. Experimental waveforms with the proposed SOC balancing method.

will be improved, but the DC-link voltage can be unstable at the end of balancing. If $P$ is decreased, the balancing speed is reduced and the SOC difference between the battery modules does not converge. Therefore, the major task is to find the proper value of $P$. In this paper, $P$ is selected as 15 through experimental trial and error so that the DC-link voltage can be stably maintained and $S O C_{\text {diff }}$ of $3 \%$ between the battery modules converges to less than $1 \%$ within 20 minutes, considering the trade-off between the balancing speed and stability. Fig. 10(a) shows the output voltage of each BPM during the equalization with the proposed SOC balancing method. In Fig. 10(a), the output voltage for each BPM is proportional to the battery voltage and SOC difference of the battery module. Fig. 10(b) shows the discharge current of each battery module, and it shows that the discharge current of each battery module 
Table 3. Comparative study of the proposed SOC balancing method.

\begin{tabular}{c|c|c|c|c|c}
\hline & {$[5]$} & {$[6]$} & {$[7]$} & {$[8]$} & $\begin{array}{c}\text { Proposed } \\
\text { method }\end{array}$ \\
\hline Auxiliary circuit for balancing & $\circ$ & $\circ$ & $\circ$ & $\mathrm{x}$ & $\mathrm{x}$ \\
\hline Capacity and type of battery & $6.2 \mathrm{Ah} / \mathrm{LiFePO} 4$ & $15.5 \mathrm{Ah} / \mathrm{Li}-\mathrm{ion}$ & $7 \mathrm{Ah} / \mathrm{Li}-\mathrm{ion}$ & $2.6 \mathrm{Ah} / \mathrm{Li}-$ ion & $200 \mathrm{Ah} / \mathrm{Lead}-\mathrm{acid}$ \\
\hline Balancing performance $(\mathrm{SOC}[\%])$ & $8.7 \% \rightarrow 0.9 \%$ & $20 \% \rightarrow 1.3 \%$ & $11.8 \% \rightarrow$ about $1.1 \%$ & $5 \% \rightarrow$ about $0.1 \%$ & $3.1 \% \rightarrow \mathrm{about} 1 \%$ \\
\hline Balancing time & $2400 \mathrm{~s}$ & $7800 \mathrm{~s}$ & $6000 \mathrm{~s}$ & $648 \mathrm{~s}$ & $960 \mathrm{~s}$ \\
\hline Balancing current & $1 \mathrm{~A}$ & $2 \mathrm{~A}$ & $0.5 \mathrm{~A}$ & $0.8 \mathrm{~A} \sim 1.6 \mathrm{~A}$ & $40 \mathrm{~A} \sim 70 \mathrm{~A}$ \\
\hline
\end{tabular}

changes in accordance with its associated output voltage of BPM. The discharge rate of the slave 2 module, which has a relatively higher $\mathrm{SOC}$, is high and the discharge rate of the master module, which has a relatively lower SOC, is low as shown in Fig. 10(b). Fig. 10(c) shows the battery voltage of each battery module during the proposed SOC balancing method. The larger ripple in the battery voltage is generated because the lead acid battery has a large internal resistance. Therefore, the battery or input voltage of each battery module is output by a digital to analog converter after using a low pass filter. The maximum initial SOC difference between the master module and the slave 2 module is approximately $3 \%$. Fig. 10 (c) also shows that the maximum initial SOC difference between the two modules converges to less than $1 \%$ about 16 minutes.

\subsection{Comparative study of the proposed SOC balancing method}

Table 3 gives a comparative study of the proposed SOC balancing method in terms of balancing speed and performance compared to the conventional method.

An accurate comparison analysis is difficult because there is no balancing algorithm with a similar condition. In [5-8], the target of SOC balancing is the cell unit of a battery that has a relatively low capacity. The balancing methods in [5-8] have a limited balancing current since it is difficult to increase the capacity of a balancing circuit built in battery management system. Therefore, if the conventional methods are applied to the high-capacity battery for EV, they are expected to exhibit a very slow balancing speed. In the method in [8], SOC balancing can be achieved without an auxiliary equalization circuit. This method also uses the battery of the cell unit, which has a relatively low capacity. The SOC balancing in [8] is implemented by a relatively high balancing current with a high C-rate (0.615), where a lithium battery is used. Therefore, it can obtain good performance in terms of balancing speed. In this paper, lead-acid batteries are used instead of lithium-affiliation batteries. Lead-acid batteries have a high internal resistance; that is, it is difficult to carry out SOC balancing with a high balancing current and a high C-rate. The maximum current and C-rate for SOC balancing in this paper are about $70 \mathrm{~A}$ and $0.35 \mathrm{C}$-rate, respectively. In this paper, the difference in the SOC value between the battery modules is about $1 \%$ after the completion of SOC balancing. The balancing performance of the proposed SOC balancing method, which represents the difference in the SOC value and voltage between battery cells or modules after completion of balancing, equates to an average in Table 3.

\section{Conclusions}

This paper proposed a control of BPMs that can achieve SOC balancing for a battery module unit without using additional balancing circuits. The SOC value of each battery module was estimated using the Coulomb counting method and battery modeling. Also, a balancing method based on the SOC of the battery has been proposed to reduce the balancing time while regulating the DC-link voltage. Simulation and experimental results were presented to demonstrate the performances of the proposed SOC balancing method. It was confirmed that the proposed SOC balancing method achieves the equalization performance in terms of the SOC difference of about $1 \%$ and the fast equalization time of about 16 minutes. Therefore, the proposed system is expected to be widely used for power converters for EVs.

\section{Acknowledgements}

This work was supported by Samsung Electronics Advanced Institute of Technology (No. IO131217-0111001).

\section{References}

[1] T.-H. Kim, J.-H. Lee and C.-Y. Won, "Design and Control methods of Bidirectional DC-DC Converter for the Optimal DC-Link Voltage of PMSM Drive", Journal of Electrical Engineering and Technology., vol. 9, no. 6, pp. 1944-1953, 2014.

[2] J.-H. Lee, D.-Y. Jung, T.-K. Lee, Y.-R and Kim, C.Y. Won, "Regenerative Current Control Method of Bidirectional DC/DC Converter for EV/HEV Application", Journal of Electrical Engineering and Technology., vol. 8, no. 1, pp. 97-105, 2013.

[3] H.-S. Park, C.-E. Kim, C.-H. Kim, G.-W. Moon and J.-H. Lee, "A Modularized Charge Equalizer for an 
HEV Lithium-Ion Battery String", IEEE Trans. Ind. Electron., vol.56, no.5 pp. 1464-1476, May. 2009.

[4] Einhorn M., Roessler W. and Fleig J., "Improved Performance of Serially Connected Li-Ion Batteries With Active Cell Balancing in Electric Vehicles", IEEE Trans. Vehi. Tech., vol. 60, no. 6, Jul. 2011.

[5] Shang Y., Zhang C., Cui N. and J. M. Guerrero, “A Cell-to-Cell Battery Equalizer With Zero-Current Switching and Zero-Voltage Gap Based on QuasiResonant LC Converter and Boost Converter", IEEE Trans. Power Electronics., vol. 30 , no. 7, pp. 37313747, July. 2015.

[6] C.-H. Kim, M.-Y. Kim, Y.-D. Kim and G.-W. Moon, "A Modularized Charge Equalizer Using a Battery Monitoring IC for Series-Connected Li-Ion Battery Strings in Electric Vehicles", IEEE Trans. Power Electron., vol. 28, no. 8, pp. 3779-3787, Aug. 2013.

[7] C.-H. Kim, M.-Y. Kim, H.-S. Park, and G.-W. Moon, "A Modularized Two-Stage Charge Equalizer with Cell Selection Switches for Series-Connected LithiumIon Battery String in an HEV", IEEE Trans. Power Electron., vol.27, no.8, pp. 3764-3774, Aug. 2012.

[8] Huang W. and Jaber A. A. Q., "Energy Sharing Control Scheme for State-of-Charge Balancing of Distributed Battery Energy Storage System", IEEE Trans. Industrial Electronics., vol. 62, no. 5, pp. 2764-2776, May. 2015.

[9] C.-S. Moo, K. S. Ng and Y.-C. Hsieh, "Parallel Operation of Battery Power Modules", IEEE Trans. Ener. Conv., vol. 23, no. 2. pp. 701-707, Jun. 2008.

[10] J.-S. Lee, J.-J. Lee, M.-R. Kim, I.-J. Park and J.-G. Kim, "Electric Model of Li-Ion Polymer Battery for Motor Driving Circuit in Hybrid Electric Vehicle", Journal of Electrical Engineering and Technology., vol. 7, no. 6, pp. 932-939, 2012.

[11] K.-C. Bae, S.-C. Choi, J.-H. Kim, Y.-C. Jung and C.-Y. Won, "LiFePO4 Dynamic Battery Modeling for Battery Simulator", IEEE International Conference on Industrial Technology(ICIT), pp. 354-358, Feb. 2014.

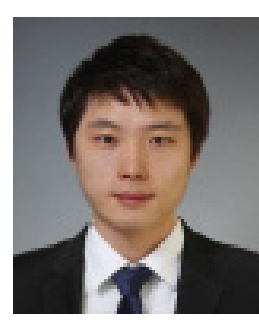

Seong-Chon Choi He received B.S. degree in electrical and electronic engineering from Kangwon University, Chuncheon, Korea in 2011, and the M.S degree in Electrical and Computer Engineering at Sungkyunkwan University, Suwon, Korea in 2013. He is currently working toward his Ph.D. degree in Electrical and Computer Engineering at Sungkyunkwan University, Suwon, Korea. His current research interests include DC/DC converters and battery management system and its robust control.

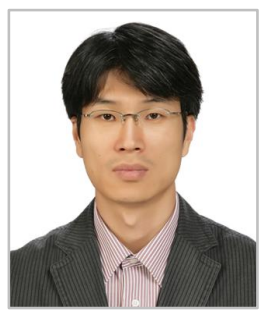

Jin-Yong Jeon He received his B.S degree in Control and Measurement engineering in 1995 and M.S degree and $\mathrm{Ph} . \mathrm{D}$ in Electronics Electrical Engineering in 2004 and 2009, respectively from University of Seoul, Seoul, South Korea. He is currently a research staff of Samsung Advanced Institute of Technology and his research interests are battery management system for electric vehicles and transverse flux linear motor control.

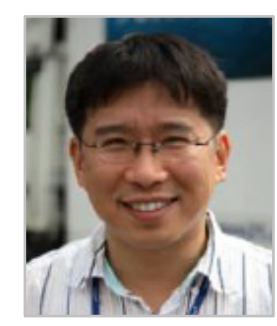

Tae-Jung Yeo He received the B.S., M.S. and Ph.D. degrees from the Department of Metallurgical Science and Engineering, Seoul National University, Seoul, South Korea, in 1993, 1995 and 2000, respectively. $\mathrm{He}$ is currently a research staff of Samsung Advanced Institute of Technology. His current research interest includes battery management technology of Electric Vehicle battery system based on intelligent data analysis.

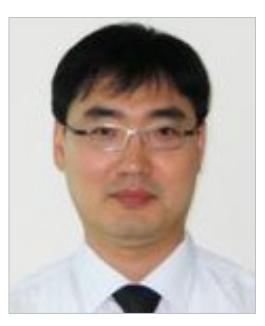

Young-Jae Kim He received the B.S. and M.S. degrees in electrical engineering from Hanyang University, Seoul, Korea, in 1998 and 2000, respectively. Since 2000, he has been with Samsung Advanced Institute of Technology, Suwon, Korea. His research interests are fuel cell system and power management system for hybrid energy system and battery management system for electric vehicles.

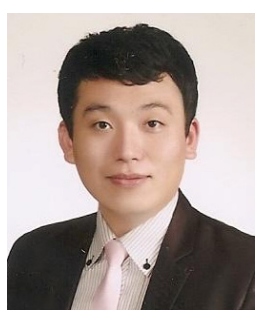

Do-Yun Kim He received B.S. degree in electrical and electronic engineering from Anyang University, Anyang, Korea in 2011, and the M.S degree in Interdisciplinary Program in Energy System Engineering at Sungkyunkwan University, Suwon, Korea in 2013. He is currently working toward his Ph.D. degree in Interdisciplinary Program in Photovoltaic System Engineering at Sungkyunkwan University, Suwon, Korea. His current research interests include inverter and battery management system and its robust control. 


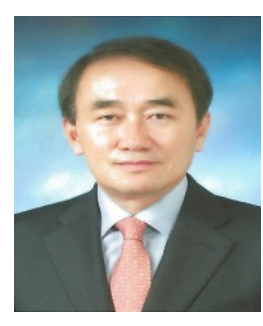

Chung-Yuen Won $\mathrm{He}$ received the B.S. degrees in Electrical Engineering from Sungkyunkwan University, Suwon, Korea, in 1978, and the M.S. and Ph.D. degrees in Electrical Engineering from Seoul National University, Seoul, Korea, in 1980 and 1987, respectively. From 1990 to 1991 , he was with the Department of Electrical Engineering, University of Tennessee, Knoxville, as a Visiting Professor. Since 1988, he has been with a member of the faculty of Sungkyunkwan University, where he is a Professor in the College of Information and Communication Engineering; also he is the director of Samsung Energy Power Research Center. He was the President of the Korean Institute of Power Electronics in 2010. Since 2011, he has been a director of the Korean Federation of Science and Technology Societies. His current research interests include the power electronic of electric machines, electric / hybrid vehicle drives, power converters for renewable energy systems. He is a senior member of the Institute of Electrical and Electronics Engineers (IEEE). 